\title{
Motor unit discharge characteristics and short term synchrony in paraplegic humans
}

\author{
N J Davey, P H Ellaway, C L Friedland, D J Short
}

\begin{abstract}
Frequency of firing and regularity of discharge of human motor units, and short term synchrony between pairs of motor units, have been assessed in extensor digitorum communis (EDC) and tibialis anterior (TA) muscles in control subjects and in clinically complete paraplegic subjects. The discharge pattern of TA motor units in paraplegia ranged from extremely regular to very irregular for different motor units whereas in the control population, and in EDC of both groups, there was a narrow, but intermediate, range of regularity. There was little difference in the incidence and degree of short term synchrony (STS) in EDC between paraplegic and normal subjects. In contrast, virtually no STS of motor units was observed in the TA muscles of the paraplegic group whereas control subjects exhibited approximately the same amount of STS in their TA and EDC muscles. It is concluded that the extra burden placed on arm muscles in paraplegia does not change the amount of synchronisation between motor units. Furthermore, section of the spinal cord does not increase STS as predicted from lesions of the reticulospinal tract in cats. This may reflect the coincidental removal of supraspinal synchronising inputs of motoneurons or the reorganisation of synaptic inputs in chronic paraplegia.
\end{abstract}

In clinically complete paraplegia there is a total loss of voluntary movement and sensory function below the level of a spinal lesion. The question here is what, over and above the loss of voluntary control, has been the consequence of interruption of the spinal cord on the characteristics of motoneuron discharge?

We have examined how motor units below the level of spinal injury differ in their discharge characteristics from those seen in the same muscles of normal subjects, and whether motor units above the level of spinal injury are affected by the lesion. Of particular interest to us is the consequence of section of descending reticulospinal pathways. In the cat, section of the reticulospinal tract descending in the dorsolateral funiculus causes irregular firing patterns and short term synchronisation (STS) of motoneuron discharges to develop. ${ }^{12}$

STS of neuronal spike discharges describes the occurrence of an impulse in one neuron within a few milliseconds of an impulse in another neuron. ${ }^{3}$ A certain amount of syn- chrony between motor unit discharges is evident in normal human subjects during a variety of motor tasks. The amount of STS varies according to the type of muscle and the task; for example, the degree of synchrony appears greater in muscles involved in performing intricate tasks ${ }^{4}$ and after training. ${ }^{5}$ Synchrony of motor unit discharge is expected to affect the quality of motor control and is likely to be a neuronal mechanism underlying tremor. ${ }^{67}$ We would predict ${ }^{12}$ that section of the dorsolateral funiculus (DLF) would create or enhance synchrony of motor unit discharge. Nevertheless, tremor is not a consistent feature of the leg musculature in paraplegia. These experiments were therefore carried out to determine the degree of STS in paraplegia and to advance our understanding of the supraspinal influences that, while not responsible for initiating motor unit discharge, may affect the pattern of discharge and precision of a motor task.

\section{Methods}

Subjects

Forty six paraplegic subjects were examined in this study. Of these, 15 provided suitable electromyographic data for the investigation (Table). Ten consented to EMG recordings being taken from muscles in both the arm (extensor digitorum communis) and the leg (tibialis anterior). These paraplegic subjects (eight male; two female; ages 18-69, mean = 36 years) were age matched with 10 healthy control subjects (five male; five female; ages $18-67$, mean $=36$ years) who had no known neurological disorder. Five paraplegic subjects consented only to recordings being made from the leg.

Ethical committee approval for this study was obtained from the Middlesex and University College Hospital Medical School, Charing Cross and Westminster Medical School and the National Spinal Injuries Centre at Stoke Mandeville. All subjects gave informed consent to the recordings after receiving a detailed explanation of the experimental procedures involved.

\section{Muscles}

Motor unit activity was examined in the tibialis anterior (TA) muscle of the leg and extensor digitorum communis (EDC) muscle of the forearm.

The TA muscle is innervated by the deep peroneal nerve which leaves the spinal cord at segment L4; all the paraplegic subjects in the study had sustained injury to their cords above this level (see table 1) and had no 
Table Details of paraplegic subjects. In subjects 1-10 EMG recordings were obtained from both EDC and TA muscles; subjects 11-15 consented to recordings only from their $T A$ muscle

\begin{tabular}{|c|c|c|c|c|c|c|}
\hline $\begin{array}{l}\text { Paraplegic } \\
\text { subject }\end{array}$ & Age/Sex (years) & $\begin{array}{l}\text { Duration of } \\
\text { paralysis }\end{array}$ & Aetiology & Neurology & Tone/Spasm & $\begin{array}{l}\text { Anti-spasticity } \\
\text { Drugs }\end{array}$ \\
\hline $\begin{array}{l}1 \\
2 \\
3 \\
4 \\
5 \\
6 \\
7 \\
8 \\
9\end{array}$ & $\begin{array}{l}18 M \\
21 M \\
22 M \\
25 M \\
26 M \\
31 M \\
41 M \\
51 F \\
53 F\end{array}$ & $\begin{array}{c}6 \mathrm{wks} \\
19 \mathrm{wks} \\
15 \mathrm{wks} \\
2 \mathrm{yrs} \\
7 \mathrm{yrs} \\
22 \mathrm{wks} \\
23 \mathrm{yrs} \\
36 \mathrm{yrs} \\
2 \mathrm{yrs}\end{array}$ & $\begin{array}{l}\text { \# T8 } \\
\# \text { T11 } \\
\# \text { L1 } \\
\# \text { T11/12 } \\
\# \text { L1 } \\
\# \text { T11/12 } \\
\# \text { T4/5 } \\
\# \text { T7/8 } \\
\# \text { T8 }\end{array}$ & $\begin{array}{l}\text { T9 C } \\
\text { L2 C } \\
\text { L3/4 C } \\
\text { T11/12 C } \\
\text { L1/2 SI/C } \\
\text { T11 C } \\
\text { T4/5 SI } \\
\text { T3 * } \\
\text { T8 SI }\end{array}$ & $\begin{array}{l}++ \\
+1- \\
+1- \\
+1- \\
+1- \\
+1- \\
++ \\
++ \\
+++\end{array}$ & $\begin{array}{l}- \\
- \\
\overline{-} \\
\overline{-} \\
\text { Diazepam } \\
\text { Diazepam } \\
\text { Dantrium and } \\
\text { Baclofen }\end{array}$ \\
\hline $\begin{array}{l}10 \\
11 \\
12 \\
13 \\
14 \\
15\end{array}$ & $\begin{array}{l}69 M \\
19 M \\
24 M \\
30 F \\
55 M \\
61 M\end{array}$ & $\begin{array}{l}13 \text { wks } \\
17 \text { wks } \\
32 \text { wks } \\
19 \text { wks } \\
23 \text { wks } \\
34 \text { yrs }\end{array}$ & $\begin{array}{l}\text { Resection aortic aneurysm } \\
\text { \# T12/L1 } \\
\# \text { T4 } \\
\# \text { T8 } \\
\# \text { T12 } \\
\text { \# T3/4 }\end{array}$ & $\begin{array}{l}\text { T7 SI } \\
\text { T11/12 C } \\
\text { T5 C } \\
\text { T9 C } \\
\text { T12 C } \\
\text { T5 SI/C }\end{array}$ & $\begin{array}{l}+1- \\
+1- \\
++ \\
+ \\
+ \\
+\end{array}$ & $\begin{array}{l}- \\
- \\
-\end{array}$ \\
\hline
\end{tabular}

Neurology

C-Complete (no motor or sensory function below lesion).

SI-Sensory Incomplete (impaired touch sensation present).

SI/C-Sensory Incomplete on admission, subsequently complete

$\star$ - Head injury and late-onset ascending lesion.

Tone/Spasm

+1 - Normal or reduced tone.

+ Slight spasms.

++ Moderate spasms
+++ Severe spasms.

voluntary control of TA. The EDC muscle is innervated by the posterior interosseus nerve which leaves the spinal cord at segment $\mathrm{C} 7$; all the paraplegics in the study had cord injuries below this level and had full voluntary control of EDC.

\section{Electromyography}

Two concentric needle electrodes (Medelec type CX-25) were placed in the muscle under study. Subjects were asked to make a weak voluntary effort in the unloaded EDC or TA muscle. The level of contraction and position of each electrode was adjusted until it was possible to distinguish the electrical profile of impulses in different, single motor units from both needle electrodes.

Paraplegic subjects had no voluntary control over their TA muscle. It was therefore necessary to elicit discharges in this muscle by reflex excitation. The procedures used were stretch of the TA muscle by extending the foot at the ankle, squeezing the achilles tendon or stroking the skin overlying the muscle. Once a discharge had been elicited it often continued for many seconds or even minutes in the absence of further stimulation or required a much reduced level of stimulation to be maintained. Occasionally, a background discharge was encountered in the absence of intentional stimulation.

Subjects were provided with feedback of muscle activity by watching the amplified impulses on an oscilloscope screen and listening to the discharge through the loudspeaker of an audio amplifier. In the EDC of both groups, and the TA of the control group, subjects were asked to use the auditory and visual feedback to maintain a constant frequency of motor unit discharge during a recording session lasting 3-4 minutes. Continuous magnetic tape recordings of motor unit discharge were made for later analysis.

While recording to tape we continuously checked that the two electrodes were recording impulses from different motor units. This was achieved by examining the timing of impulses in the two trains on a dual-beam oscilloscope.

Cross correlation of motor unit discharge

Motor unit impulses were digitised for time series analysis by computer. Several 10 s periods of continuous discharge from a pair of motor units were used to construct a crosscorrelogram. ${ }^{89}$ The location of a peak of short term synchrony (STS) in a cross-correlogram was judged by eye and by use of the cumulative sum derivative (cusum) of the correlogram. ${ }^{10}$ To construct the cusum the mean count $(m)$ in a number of correlogram bins was calculated in a region not associated with any primary peak or secondary feature of the correlogram. This was usually taken as a span of 90 bins, each $1 \mathrm{~ms}$ in duration, ending at least $30 \mathrm{~ms}$ before the time of a reference impulse (bin zero). The accumulated summed differences between this mean and the individual bin counts represents the cusum. A peak in the correlogram, signalling an increased probability of discharge of one motor unit given the discharge of the other, was accepted as significant $(\mathrm{p}<0.001)$ if the total number of counts in the $n$ bins constituting the peak exceeded the counts expected by chance alone $(\mathrm{nm})$ by more than $3.29 \sqrt{ } \mathrm{nm}^{89}$

A quantitative estimate of the correlation during short term synchrony was calculated as the likelihood (above chance) of discharge of one motor unit (train A) given an impulse in the other motor unit (train B), ${ }^{11}$ and vice versa. These values are represented by the excursion of the cusum in the region of the primary peak of the cross-correlogram. The two indices produced are given the abbreviations XP A/B and XP B/A.

The incidence of synchrony was calculated as the percentage of motor unit pairs showing 
significant peaks of synchrony in a particular muscle for each subject. This figure was then averaged for a number of subjects to estimate the incidence of synchrony within a particular experimental group.

Frequency and regularity of discharge

Frequency of discharge of a motor unit was taken as the mean rate calculated during a stationary period of discharge. During weak voluntary contraction one unit was almost invariably discharging at its recruitment frequency and this usually applied to the other unit as recruitment thresholds were similar.

The regularity of discharge of an individual motor unit was assessed by inspection of the auto-correlogram function and by measuring the coefficient of variation ( $V$ ) of the interspike time intervals during stationary periods of discharge. ${ }^{1213} \mathrm{~V}$ is calculated as the standard deviation of the distribution of interspike time intervals divided by the mean interspike interval. Stationarity of the mean rate of discharge was determined by inspection of a sequential display of interspike time intervals and the rejection of periods comprising trends in these intervals.

\section{Results}

Cross-correlation analysis

In 10 paraplegic subjects, discharges from a total of 38 EDC motor units were recorded; from these 22 cross-correlograms were constructed between different pairs of motor units. In the same subjects, discharges were recorded from 44 TA motor units and crosscorrelations were assessed for 22 different pairs. In 10 age-matched control subjects, 41 cross-correlograms were constructed from the discharges of 58 EDC motor units and 42 correlations were computed from 62 TA motor units. Cross correlograms and cusum derivatives constructed from EDC and TA motor units that displayed STS in a control subject are presented in fig 1. The correlograms for both the EDC and TA muscle (fig 1A, B) show peaks at time zero indicating STS with a duration of 9-15 ms. The correlogram in Fig 1C is constructed from motor units recorded from the EDC muscle in a paraplegic subject (number 6, table). STS is evident as a peak about time zero in the correlogram. In contrast, a pair of motor units in the TA muscle of the same paraplegic subject showed no STS (fig 1D).

STS was frequently observed between the discharges of motor units in EDC and TA of normal subjects and EDC of paraplegics but was rarely encountered in the TA of paraplegics. The actual incidence (see methods) of short term synchrony (fig $2 \mathrm{~A}$ ) between the discharges of motor units in EDC muscles was $63 \%$ in control subjects and $77 \%$ in paraplegic subjects. A similar proportion $(66 \%)$ of the pairs of TA motor units in the control subjects exhibited a significant degree of synchrony. However, of the 22 cross-correlations between TA motor units in paraplegic subjects, only one (number 5, table) showed a significant peak (fig 3B) and there is doubt as to whether this represents STS since the total counts in the
Figure 1 Crosscorrelograms (top) and their cumulative sum derivatives (bottom) constructed from the discharges of pairs of motor units in a control subject ( $A: E D C$ muscle, $B: T A$ muscle) and $a$ paraplegic subject (number 6, table) (C: EDC muscle, D: TA muscle). Ordinates: probability of a spike in each $1 \mathrm{~ms}$ bin, given a reference spike in the other motor unit at time zero (cross correlogram), and deviation from the expected cumulative probability (cusum). The correlograms are constructed from between 80 and 240 s of motor unit discharge. Note the absence of synchrony at time zero in the $T A$ muscle of the paraplegic subject.

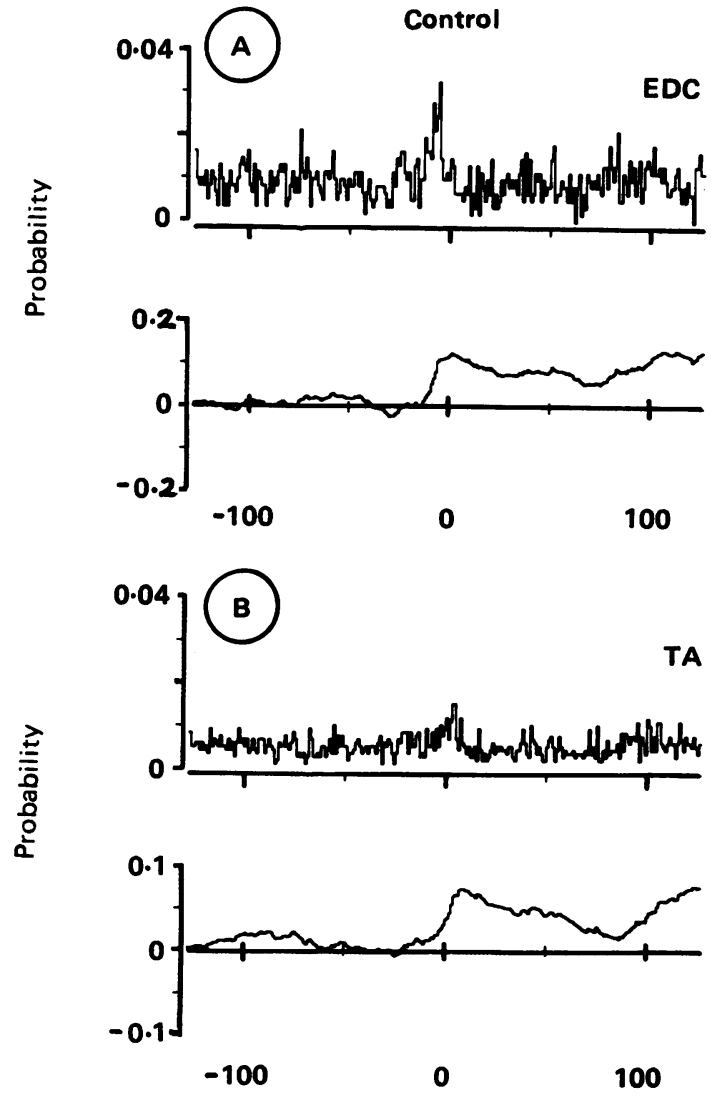

Time (ms)
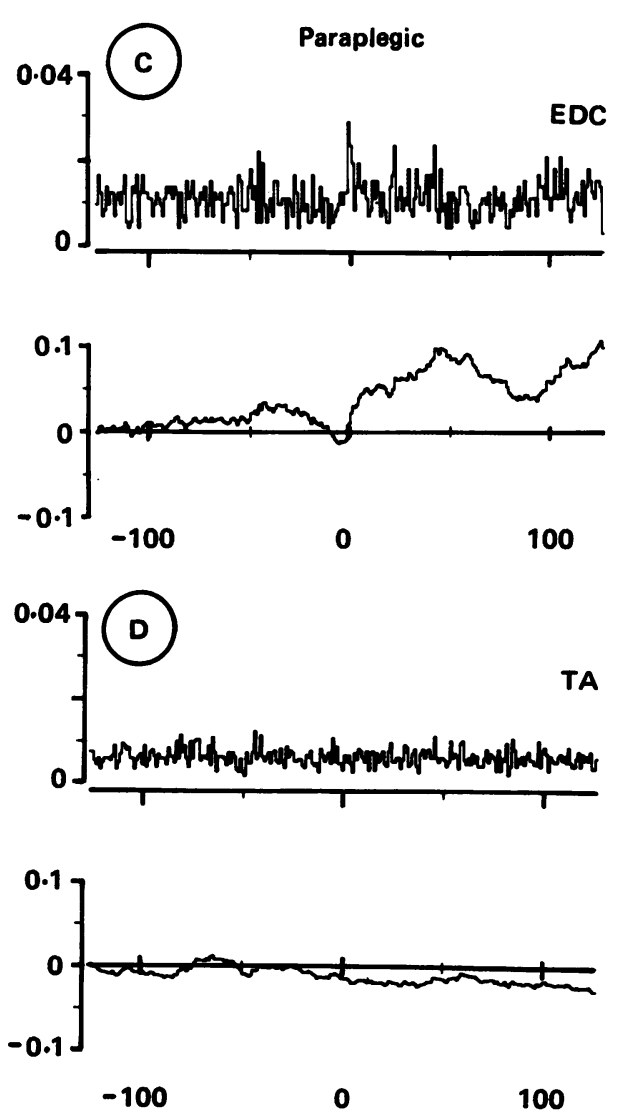

Time (ms) 
Figure 2 A: Incidence of short term synchrony of motor unit discharge in $T A$ and EDC muscles of control and paraplegic subjects. B: Distribution of degree of synchrony of motor unit discharge in the EDC muscle (left) and TA muscle (right) of control (open bars) and paraplegic (hatched bars) subjects. Degree of synchrony $(X P)$ is expressed as the likelihood (above chance) of a spike in one motor unit given a spike in the other motor unit.

Figure 3 Crosscorrelograms (above) and cusums (below) constructed from the discharges of motor units recorded in paraplegic subjects. Ordinates as in Fig 1A: EDC motor units. Short term synchrony is present at time zero. $B$ : $T A$ motor units in the same subject (number 5 , table) as A. Suspected synchrony at time zero. $C$. The other example of a significant peak in a crosscorrelogram constructed from the discharges of $T A$ motor units in a paraplegic subject (number 15, table).
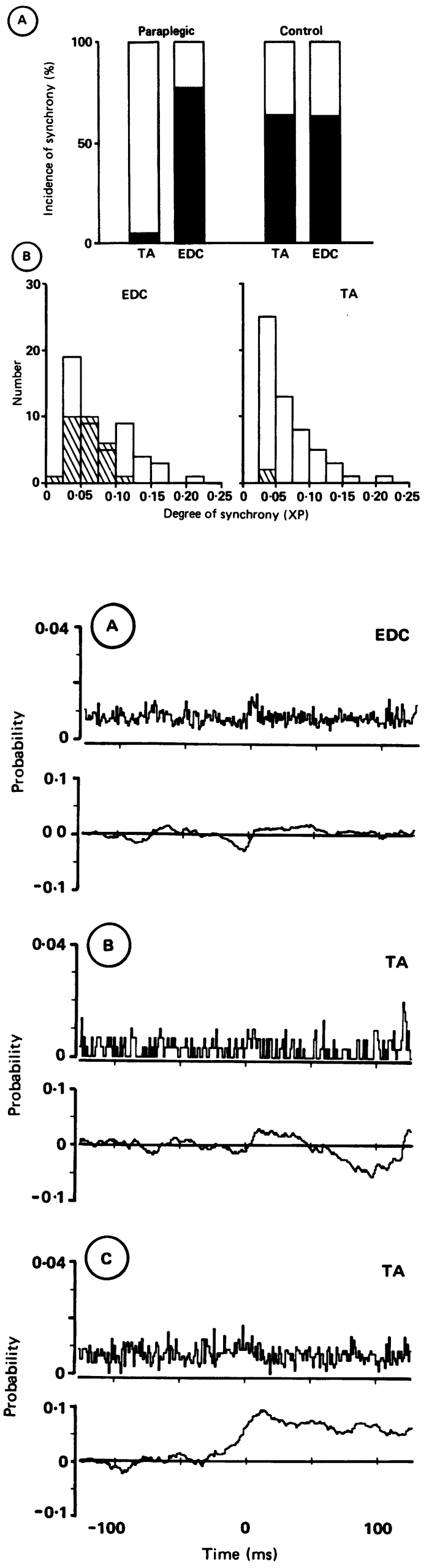

correlogram were low and a peak at a time other than zero was also present. The same paraplegic subject exhibited a moderate amount of STS in the EDC muscle (fig 3A).

In those cases where a peak was evident in a correlogram the amount of STS was assessed (see Methods) and expressed as the likelihood (above chance) of firing of one motor unit given a discharge in the other motor unit. As an example, the values for the STS seen in fig $1 \mathrm{~A}$ are, for the likelihood of an A spike given a B spike (XP A/B) 0.105 and for a B spike given an A spike (XP B/A) 0.091. That is, synchrony above that expected by chance occurred for approximately one in ten of the spikes of both motoneurons.

The distributions of XP values for control and paraplegic subjects are shown in fig 2 . In the EDC muscle the range of degree of synchrony (XP) in control subjects is 0.03 to 0.204 (mean $=0.08$ ) and in the paraplegic group is 0.02 to 0.11 (mean $=0.06$ ). although the mean values are similar, it should be noted that 17 of the 50 values of XP in the control group are above 0.10 whereas this proportion is only one out of 28 in paraplegic subjects, indicating less synchrony in the EDC of paraplegic subjects.

The amount of synchrony between pairs of TA motor units in control subjects ranged from 0.03 to 0.21 (mean $=0.08$ ) which is a similar range to that seen in control EDC muscles. The single significant correlogram peak between TA motor units in paraplegic subjects (fig 3B) had synchrony indices of 0.045 and 0.035 . These values fell at the lower end of the range of values assessed for TA units in normal subjects.

Recordings were made solely from the TA muscle in a further five paraplegic subjects. Nineteen motor units were recorded and 14 different correlograms constructed. One correlogram (subject 15, table) exhibited a broad increase in level of counts around time zero which was not typical of the STS seen in normal subjects (fig 3C). Nevertheless, the indices of synchrony were calculated for the central bins. The resulting values $(0.024$ and 0.052 ) were low compared with the range seen in the control group (fig 2B).

\section{Discharge rates and variability}

Discharge frequencies of motor units during sustained weak contraction of the EDC muscle of control subjects ranged from 8.5 to 15.8 ips (mean $=11.8 \mathrm{ips}$ ). Discharge rates in EDC during similar voluntary contractions by paraplegic subjects ranged between 8.5 and $17 \cdot 3$ (mean $=13.1 \mathrm{ips}$ ). The small difference in mean rates was statistically significant (Student's $t$ test, $\mathrm{p}<0.001)$.

Firing frequencies of TA motor units in control subjects ranged from 6.6 to 11.4 ips (mean $=8.9 \mathrm{ips}$ ). In paraplegics, the reflexly induced discharges of TA motor units had a wider range of firing frequencies $(1.7$ to 21.5 , mean $=7.5$ ips) than those measured during weak voluntary effort in control subjects. The broad range of discharge frequencies seen in the TA muscle of paraplegics may have been due, in part, to the lack of uniformity of the 
Figure 4 The relation between regularity of discharge and frequency of discharge in $T A$ motor units in control (open circles) and paraplegic (closed circles) subjects. Points for motor units with the highest and lowest frequency of discharge for each subject are presented. Regularity of discharge is expressed as coefficient of variation $(V)$ of interspike intervals.

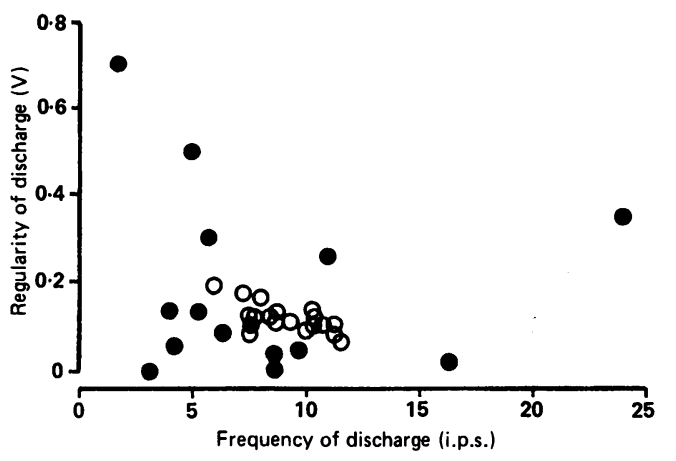

stimuli used to excite the motor units.

Regularity of discharge, expressed as coefficient of variation (V) of the interspike intervals (see Methods), was measured during stationary periods of discharge. In EDC V ranged from 0.05 to 0.26 (mean $=0.12$ ) in control subjects and from 0.07 to 0.20 (mean $=$ $0 \cdot 13$ ) in paraplegics. In the TA muscle $\mathrm{V}$ ranged from 0.04 to 0.20 (mean $=0.11$ ) in control subjects and had a similar spread to that seen in EDC. In contrast regularity of motoneuron discharge in paraplegic subjects had a much wider range $(0.003$ to 0.71 , mean $=$ $0 \cdot 14)$.

Regularity (V) of TA motor unit discharges for both control and paraplegic subjects is plotted against discharge frequency in fig 4 . It is evident that in paraplegic subjects the range of regularity seen below the site of injury in the TA muscle is much broader than in the TA muscles of control subjects. It is also apparent (fig 4) that some motor units from TA muscles in paraplegics can be extremely regular in their discharges $(\mathrm{V}<0.01)$. This highly regular discharge pattern is apparently not dependent on firing frequency and is associated both with motor units having low and high rates of discharge.

\section{Discussion}

The incidence of synchrony in the EDC muscle of the forearm of paraplegic and control subjects has been found to be similar. This suggests that the spinal cord trauma, drug therapy and lifestyle changes adopted by paraplegics has not affected the tendency to motoneuron synchrony in muscles above the level of spinal injury. However, in control subjects $34 \%$ of the estimates of degree of synchrony in EDC lay above $0 \cdot 1(\mathrm{XP})$ whereas only 1 value $(3.6 \%)$ was greater than 0.1 in paraplegics. The absence of higher degrees of synchrony in the EDC of paraplegics may have resulted from loss of shared synaptic inputs to brachial motoneurons from ascending propriospinal neurons. ${ }^{14}$

A marked difference in the tendency to synchrony in the TA muscle was observed between paraplegic and normal control subjects. Only one doubtful instance of synchrony was seen in the TA muscle of the main group of paraplegic subjects compared with an incidence of $66 \%$ in normal subjects. Our prediction from studying synchrony of motoneuron discharge in the cat ${ }^{2}$ is that severance of reticulo-spinal tracts which run in the dorsolateral funiculus (DLF) would result in more, not less, synchrony. Why, then, is motor unit synchrony not evident below the site of spinal injury in paraplegia when, presumably, the reticulospinal pathways are inoperative?

Patients with no voluntary motor control and no sensation below their injury site are assumed to have complete lesions as assessed clinically. ${ }^{15}$ In such patients descending pathways that are not directly involved in the recruitment of motoneurons may persist and remain undetected. Necropsy examination of the spinal cord of paraplegics who had been diagnosed as having "clinically complete" lesions has revealed anatomical continuity in some nerve fibres. ${ }^{16}$ Dimitrejevic et $a l^{17}$ have been able to activate muscles below the site of a "clinically complete"' lesion by reinforcement manoeuvres above the injury site. They suggest that such activation provides evidence for persistent functional connections in descending pathways. Descending reticulospinal fibres that have survived may therefore have been actively inhibiting segmental inputs capable of synchronising TA motor unit discharges in paraplegic subjects. It seems unlikely, however, that the DLF would have been spared in all the paraplegic subjects that we have studied.

The relative paucity of synchrony in the TA muscle of paraplegic subjects may also be the result of a loss of shared input to motoneurons from supra-spinal sources. A study of motoneuron synchrony in deafferented humans ${ }^{18}$ revealed no difference in motoneuron synchronisation from that expected in normal subjects. This result reinforced earlier studies ${ }^{4}$ suggesting that in humans corticospinal fibres can supply afferent input capable of synchronising motoneurons.

A further factor which may account for the difference in the incidence of synchrony following acute spinal section in the cat ${ }^{12}$ and that observed in paraplegic humans is the time delay between the trauma and assessment of synchrony. In cats, synchrony between motoneurons is seen to develop within an hour of acute spinal cord lesion and lasts for at least 24 hours. Our recordings in paraplegic subjects were made a minimum of six weeks or, in most cases, many months or years after their accident. For some time after a mechanical insult to the spinal cord reflexes below the injury site are substantially suppressed. ${ }^{19}$ Spinal shock is not a static phenomenon ${ }^{20}$ and, following the period of depression, recovery and hyperexcitability of reflexes occurs. Collateral sprouting of intact axons takes place $^{21}$ and is likely to change the balance of synaptic inputs to motoneurons. Shared synaptic inputs capable of having a synchronising action may thus form a proportionally smaller part of the newly organised synaptic inputs to reinnervated motoneurons.

It should also be borne in mind that many of the motor unit discharges recorded from the TA muscles of paraplegic subjects were elicited and, in some cases, maintained by innocuous stimulation of the legs. Stimulation included rubbing the skin overlying the TA muscle, 
stretching the muscle or massaging the tendon (see methods). Such stimuli do not cause prolonged discharge of motor units in normal subjects so a comparable study on the influence of cutaneous and proprioceptive input on motoneuron discharge synchrony in normal and paraplegic humans could not be carried out. However, we have no reason to suspect that the increased segmental afferent input would decrease the amount of short term synchrony since in cats the reverse is the case. ${ }^{9}$

The discharge pattern of motor units in the TA muscle in paraplegics differs substantially from muscles above the injury and from muscles in control subjects. The motor units have a wider range of discharge interval variability and firing frequency. Some units are characteristically very regular and have rather low discharge frequencies. This is unusual since low firing rates have been associated with greater variability in interspike intervals. ${ }^{21422}$ Some of these highly regular motoneurons were firing spontaneously in the absence of intentional stimulation. This unusually regular discharge may represent a pathological condition of the motoneuron when the principal depolarising component has an intrinsic rather than synaptic source. Other TA motor units were considerably more irregular in their discharges than normal which, considering that motoneurons have a pronounced period of hyperpolarisation following a spike, suggests that they were subjected to synaptic inputs that cause large post synaptic potentials. If these inputs were shared between motoneurons then they would be expected to cause synchronisation. ${ }^{2}$ However, no evidence of synchrony was seen when the discharges of two of the less regular motor units were cross-correlated. We conclude therefore that the discharge pattern is a result of the pathological condition of the motoneurons.

In conclusion, we have shown that motor unit synchrony is either weak or absent in the TA muscle of paraplegics and suggest therefore that short term synchrony is not a contributing factor to abnormal motor activity in paraplegia.

We are grateful for the help and support from the staff and patients at the National Spinal injuries Unit, Stoke Mandeville Hospital. In particular, we would like to thank Drs Frankel, Jamous, Silver, Vernon and $\mathbf{M r}$ Gardner for their encouragement and the patients for always being ready and willing to assist us with this research. The work was supported by a grant from the Wellcome Trust. CLF is a SERC Scholar.

1 Connell LA, Davey NJ, Ellaway PH. The degree of short term synchrony between alpha and gamma motoneurones coactivated during the flexion reflex in the cat. $J$ Physio 1986;376:47-62.

2 Davey NJ, Ellaway PH. Control from the brain stem of synchrony of discharge between gamma motoneurones in the cat. Exp Brain Res 1988;72:249-63.

3 Sears TA, Stagg D. Short term synchronization of intercostal motoneurone activity. J Physiol 1976;263:357-87.

4 Datta AK, Stephens JA. Short term synchrony of motor unit firing in human first dorsal interosseus muscle. $J$ Physio 1980;308:19-20.

5 Milner-Brown HS, Stein RB, Lee RG. Synchronisation of human motor units: possible roles of exercise and supraspinal reflexes. Electroenceph Clin Neurophysiol 1975;38:245-54.

6 Taylor A. The significance of grouping of motor unit activity. J Physiol 1962;162:259-69.

7 Allum JH, Dietz V, Freund H-J. Neuronal mechanisms underlying physiological tremor. J Neurophysiol 1978;41:557-71.

8 Ellaway PH, Murthy KSK. The origins and characteristics of cross-correlated activity between gamma motoneurones in the cat. $Q J \operatorname{Exp}$ Physiol 1985;70:219-32.

9 Ellaway PH, Murthy KSK. The source and distribution of short term synchrony between gamma motoneurones in the cat. $Q J$ Exp Physiol 1985;70:233-47.

10 Davey NJ, Ellaway PH, Stein RB. Statistical limits for detecting change in the cumulative sum derivative of the peristimulus time histogram. J Neurosci Meth 1986;17:153-66.

11 Davey NJ, Ellaway PH, Friedland CL. Quantitative assessment of the degree of synchrony between the discharges of neurones. J Physiol 1987;390:10.

12 Matthews PCB, Stein RB. The regularity of primary and secondary muscle spindle afferent discharges. J Physiol 1969;202:59-82.

13 Ellaway PH. The variability in discharge of fusimotor neurones in the decerebrated cat. Exp Brain Res 1972;14:105-17.

14 Miller S, Reitsma DJ, Van der Meche FGA. Functional organisation of the long ascending propriospinal pathways linking lumbosacral and cervical segments in the cat. Brain Res 1973;62:169-88.

15 Frankel HL, Hancock DO, Kyslop J, et al. The value of postural reduction in initial management of closed injuries of the spine and with paraplegia and tetraplegia, Part I. Paraplegia 1969;7:179-92.

16 Kakulas BA, Bedbrook GM. Pathology of injuries of the vertebral spinal cord-with emphasis on the macroscopic aspects. In: Vinken PJ, Bruyn GW, eds. Handbook of aspects. In: Vinken PJ, Bruyn GW, eds. Handbook of
Clinical Neurology, Vol. 25. Amsterdam: North-Holland, Clinical Neurolo

17 Dimitrijevic MR, Dimitrijevic MM, Faganel J, Sherwood AM. Suprasegmentally induced motor unit activity in paralysed muscles of patients with established spinal cord injury. Ann Neurol 1984;16:216-21.

18 Baker JR, Bremner FD, Cole JD, Stephens JA. Short-term synchronization of intrinsic hand muscle motor units in a deafferented man. J Physiol 1988;396:155.

19 Fulton JF, McCouch GP. The relation of motor area of primates to the hypereflexia (spinal shock) of spinal primates to the hypereflexia (spinal shock)
transection. J Nerv Ment Dis 1937;86:125-46.

20 Weller RO, Illis LS. Damage to the nervous system. In: Illis LS, Sedgewick EM, Glanville HJ, eds. Rehabilitation of the neurological patient. Oxford: Blackwell 1982:13-43.

$21 \mathrm{Liu}$ CN, Chambers WW. Collateral sprouting in the spinal cord. In: Windle WF, eds The Spinal cord and its reaction to trauma. New York: Marcel Dekker Inc, 1982:219-36.

22 Stein RB. A theoretical analysis of neuronal variability. Biophysics J 1965;5:173-94. 